\title{
STRUCTURE OF THE SOLUTION SET OF SOME FIRST ORDER DIFFERENTIAL EQUATIONS OF COMPARISON TYPE
}

\author{
BY \\ T. G. HALLAM( $\left.{ }^{1}\right)$ AND J. W. HEIDEL $\left({ }^{2}\right)$
}

\begin{abstract}
The comparison principle is a powerful tool that has a wide variety of applications in ordinary differential equations. The results of this article describe the geometric structure of the solution space of some first order scalar differential equations that may arise in the comparison method. A quite general class of differential equations is found to have a similar solution set configuration as the differential equation of separable variable type. One of the main results establishes, under certain conditions, that there is a unique unbounded solution of the first order differential equation which exists on an interval of the form $\left[t_{0}, \infty\right)$. Furthermore, this unbounded solution separates the solutions that are bounded on $\left[t_{0}, \infty\right)$ from those that are not continuable to all $t>t_{0}$.
\end{abstract}

1. Introduction. In recent years the comparison method for the study of stability and asymptotic behavior of the solutions of ordinary differential equations has been extensively developed [10], [11]. The importance of this method lies in the fact that properties of the solution set of systems of ordinary differential equations can be inferred from corresponding properties of the solution set of a scalar differential equation. This scalar comparison equation is usually of the form

$$
d r / d t=w(t, r)
$$

where $w=w(t, r)$ is continuous and nonnegative for $t \geqq 0$ and $r>0$.

If (1.1) is of variables separable type, that is, of the form

$$
d r / d t=q(t) K(r),
$$

then the structure of the solution set can readily be determined (see the discussion below). It is the purpose of this article to study the asymptotic structure of the solution set of (1.1) when it is not necessarily solvable. This is accomplished by showing that the solutions of very general equations (1.1) have the same structure

Received by the editors August 31, 1970 and, in revised form, January 5, 1971.

AMS 1969 subject classifications. Primary 3401, 3440; Secondary 3442, 3450, 3453, 3490.

Key words and phrases. Comparison principle, separation of variables, asymptotic behavior, solution set configuration.

(1) The research of this author was supported in part by the National Science Foundation under grant GP-11543.

${ }^{2}$ ) The research of this author was supported in part by a University of Tennessee Faculty Research Grant.

Copyright (C) 1971, American Mathematical Society 
as those of (1.2). Thus, it is desirable to briefly indicate what happens in the separable case (1.2). For additional details, refer to [1]. Assume now that $q=q(t)$ is nonnegative and continuous for $t \geqq 0$ and $K=K(r)$ is positive and continuous for $r>0$. The four possible combinations of convergence and/or divergence of the improper integrals $\int^{\infty} q(\tau) d \tau$ and $\int^{\infty}[1 / K(\rho)] d \rho$ lead to the four different solution set configurations which are indicated below.

Configuration 1. If

$$
\int^{\infty} q(\tau) d \tau<\infty \text { and } \int^{\infty}[1 / K(\rho)] d \rho<\infty,
$$

then there exists a unique unbounded solution $\psi=\psi(t)$ of (1.2). This solution $\psi$ separates the bounded solutions of (1.2) from the solutions of (1.2) with a finite escape time; that is, if $r=r(t)$ is a solution of (1.2) with $0<r\left(t_{0}\right)<\psi\left(t_{0}\right)$ then $r$ is bounded on $\left[t_{0}, \infty\right)$, and if $r\left(t_{0}\right)>\psi\left(t_{0}\right)$ there exists a $T\left(t_{0}<T<\infty\right)$ such that $\lim _{t \rightarrow T^{-}} r(t)=\infty$.

Configuration 2. If

$$
\int^{\infty} q(\tau) d \tau<\infty \text { and } \int^{\infty}[1 / K(\rho)] d \rho=\infty
$$

then all solutions of (1.2) are bounded.

Configuration 3. If

$$
\int^{\infty} q(\tau) d \tau=\infty \text { and } \int^{\infty}[1 / K(\rho)] d \rho<\infty
$$

then all solutions of (1.2) have a finite escape time.

Configuration 4. If

$$
\int^{\infty} q(\tau) d \tau=\infty \text { and } \int^{\infty}[1 / K(\rho)] d \rho=\infty
$$

then all solutions of (1.2) are continuable to the right and are unbounded on $[0, \infty)$.

$\$ 3$ of this paper shows that each of these configurations is valid for a large class of equations of the form (1.1) which are not, in general, solvable in a closed form. In order to accomplish this, it is useful to prescribe a certain monotonicity property on $w$. Namely, we postulate the existence of a function $L_{w}(r)$ such that the function $w(t, r) L_{w}(r)$ may be written as the sum of a nondecreasing and a nonincreasing function of $r$. This type of assumption is consistent with a recent series of papers [2], [4], [9] on the asymptotic behavior of systems of differential equations where it is required that $w=w(t, r)$ be nondecreasing in $r$ for $r>0$ and fixed $t$.

The comparison technique may also lead to the scalar equation

$$
d r / d t=-w(t, r)
$$


where $w$ satisfies the above-mentioned conditions (see [1], [3]). Here, one case of interest is when $w=w(t, r)$ is nonincreasing in $r$ for $r>0$ and each fixed $t$ [3]. In $\S 4$ it is shown how the structure of the solution set for (1.3) can be determined by transforming (1.3) into the form of (1.1) and using the results of $\S 3$. In particular, the case when $w$ (of (1.3)) is nonincreasing in $r$ is discussed.

In order that a scalar equation be of value for use in the comparison principle, it must have bounded (positive) solutions. When $w=w(t, r)$ is nondecreasing in $r$ for $r>0$ and each fixed $t$, it turns out that the condition $\int^{\infty} w(\tau, \lambda) d \tau<\infty$ for some $\lambda>0$ is necessary and sufficient for the existence of bounded solutions of (1.1) [4]. Therefore, for the purposes of the comparison technique, the Configurations 1 and 2 are the important ones. Most of our effort in $\$ 3$ is devoted to a discussion of the first solution set configuration since it is the most complicated. The other three solution set configurations are comparatively easy to handle and are dealt with primarily for completeness.

It is perhaps worth mentioning another broad class of scalar equations, the Riccati and generalized Riccati equations, which is generally distinct from the comparison-type equation considered here. In this case, one generally does not have $\left|\int^{\infty} w(\tau, \lambda) d \tau\right|<\infty$ for any $\lambda>0$. However, the basic problem of interest here is to find conditions which imply the existence, or nonexistence, of positive continuable solutions. Some papers which consider generalized Riccati equations are [5], [6], [7], and [8].

2. Basic hypotheses. With respect to equations (1.1) and (1.3) it will be assumed that $w=w(t, r)$ is continuous and nonnegative on $[0, \infty) \times(0, \infty) . L_{w}(r)$ is a continuous, positive function defined for $r>0$ and is chosen so that $w$ can be decomposed as follows:

$$
w(t, r)=w_{1}(t, r)+w_{2}(t, r)
$$

where

$w_{1}(t, r) L_{w}(r)$ is nonnegative and nondecreasing in $r$ for $r>0$ and each fixed $t \geqq 0$;

and

$w_{2}(t, r) L_{w}(r)$ is nonnegative and nonincreasing in $r$ for $r>0$ and each fixed $t \geqq 0$.

Sometimes, it will be necessary to impose the following conditions:

Let there exist a continuous function $p_{1}(t)$ defined on $[0, \infty)$ so that $w_{1}(t, r) L_{w}(r) \leqq p_{1}(t)$ for all $r>0, t \geqq 0$.

Let there exist a continuous function $p_{2}(t)$ defined on $[0, \infty)$ so that

$w_{2}(t, r) L_{w}(r) \geqq p_{2}(t)$ for all $r>0, t \geqq 0$. Furthermore, for each $t \geqq 0$, if $w_{2}(t, r)>0$ for $r>0$ then $p_{2}(t)>0$. 
It will always be assumed that equations (1.1) and (1.3) have a unique solution to the initial value problem. The unique solution of (1.1) or (1.3) which passes through the point $\left(t_{0}, r_{0}\right)$ will be designated by $r\left(t ; t_{0}, r_{0}\right)$. The reciprocal of $L_{w}(r)$ will be denoted by $L_{w}^{-1}(r)$.

3. Results for equation (1.1). The results in this section are valid for equation (1.1) subject to the requirements imposed in the previous section. The conclusions are reached by an investigation of the convergence or divergence of the integrals

$$
\int^{\infty} w(\tau, \lambda) d \tau \text { and } \int^{\infty} L_{w}(\rho) d \rho .
$$

The first result, Theorem 1, shows that the solution set of a very general equation has almost the same structure as Configuration 1. Theorem 2 then shows that some additional conditions imply the existence of a unique "separatrix" solution, that is, a solution which separates the bounded solutions from the solutions with finite escape time. This is precisely the structure of Configuration 1.

THEOREM 1. Let (2.1), (2.2), and (2.4) be satisfied; furthermore, suppose that $w \not 0$ for large $t$,

$$
\int^{\infty} w(\tau, \lambda) d \tau<\infty
$$

for all $\lambda>0$, and

$$
\int^{\infty} L_{w}(\rho) d \rho<\infty
$$

Then, there exist nonnegative constants $t_{0}, \alpha, \beta$ with $0<\alpha \leqq \beta<\infty$ such that if $r_{0}$ satisfies the inequality

(i) $0<r_{0}<\alpha$ then $r\left(t ; t_{0}, r_{0}\right)$ is bounded on $\left[t_{0}, \infty\right)$; hence $\lim _{t \rightarrow \infty} r\left(t ; t_{0}, r_{0}\right)$ exists and is positive.

(ii) $\alpha \leqq r_{0} \leqq \beta$ then $r\left(t ; t_{0}, r_{0}\right)$ exists on $\left[t_{0}, \infty\right)$ and $\lim _{t \rightarrow \infty} r\left(t ; t_{0}, r_{0}\right)=\infty$.

(iii) $\beta<r_{0}$ then $r\left(t ; t_{0}, r_{0}\right)$ is not continuable to all $t>t_{0}$; hence, there exists $a$ $T_{0}>t_{0}$ such that $\lim _{t \rightarrow T_{0}^{-}} r\left(t ; t_{0}, r_{0}\right)=\infty$.

Proof. First, the condition (3.1) implies that there exists a bounded solution of (1.1). Let $r_{0}>0$ be given; select $T_{0}=T_{0}\left(r_{0}\right)>0$ so that

$$
2 \int_{T_{0}}^{\infty} w_{1}\left(\tau, 2 r_{0}\right) d \tau<L_{w}^{-1}\left(2 r_{0}\right) \int_{r_{0}}^{2 r_{0}} L_{w}(\rho) d \rho
$$

and

$$
2 \int_{T_{0}}^{\infty} w_{2}\left(\tau, r_{0}\right) d \tau<L_{w}^{-1}\left(r_{0}\right) \int_{r_{0}}^{2 r_{0}} L_{w}(\rho) d \rho .
$$

For any $t_{0} \geqq T_{0}$, we claim that $r\left(t ; t_{0}, r_{0}\right)<2 r_{0}$. For, if this is false, then there exists a first $t_{1}>t_{0}$ where $r\left(t_{1} ; t_{0}, r_{0}\right)=2 r_{0}$ and $r\left(t ; t_{0}, r_{0}\right)<2 r_{0}, t \in\left[t_{0}, t_{1}\right)$. 
From (1.1), (2.1) and (2.2), we obtain

$$
\begin{aligned}
\int_{r_{0}}^{2 r_{0}} L_{w}(\rho) d \rho & =\int_{t_{0}}^{t_{1}} w\left(\tau, r\left(\tau ; t_{0}, r_{0}\right)\right) L_{w}\left(r\left(\tau ; t_{0}, r_{0}\right)\right) d \tau \\
& \leqq L_{w}\left(2 r_{0}\right) \int_{t_{0}}^{t_{1}} w_{1}\left(\tau, 2 r_{0}\right) d \tau+L_{w}\left(r_{0}\right) \int_{t_{0}}^{t_{1}} w_{2}\left(\tau, r_{0}\right) d \tau \\
& <\int_{r_{0}}^{2 r_{0}} L_{w}(\rho) d \rho .
\end{aligned}
$$

This contradiction establishes that there exists a bounded solution of (1.1). Hence, we take $t_{0}$ in the conclusion of the theorem as $T_{0}$ and by defining

$$
\alpha=\sup \left\{r_{0}: r\left(t ; t_{0}, r_{0}\right) \text { is bounded on }\left[t_{0}, \infty\right)\right\},
$$

we then have $\alpha>0$.

A solution with a finite escape time will now be exhibited. Define the function $G(r)=-\int_{r}^{\infty} L_{w}(\rho) d \rho$ for $r>0$. Using hypothesis (2.1) and (2.4), we obtain

$$
r\left(t ; t_{0}, r_{0}\right) \geqq G^{-1}\left[G\left(r_{0}\right)+\int_{t_{0}}^{t} p_{2}(\tau) d \tau+L_{w}\left(r_{0}\right) \int_{t_{0}}^{t} w_{1}\left(\tau, r_{0}\right) d \tau\right]
$$

where $G^{-1}$ is the inverse of $G$ and for all $t$ for which the right side of (3.3) makes sense. Because $G\left(r_{0}\right)<0$ and not both $w_{1}$ and $p_{2}$ are identically zero for large $t$, numbers $T, t_{1}$, and $r_{0}$ may be found so that

$$
G\left(r_{0}\right)+\int_{t_{1}}^{T} p_{2}(\tau) d \tau+L_{w}\left(r_{0}\right) \int_{t_{1}}^{T} w_{1}\left(\tau, r_{0}\right) d \tau=0
$$

Therefore, there exists a $T_{1} \leqq T$ so that $\lim _{t \rightarrow T_{1}^{-}} r\left(t ; t_{1}, r_{0}\right)=\infty$. In fact, given any $t_{1}>0$, it is possible to choose $r_{0}$ sufficiently large so that

$$
-G\left(r_{0}\right)<\int_{t_{1}}^{\infty} p_{2}(\tau) d \tau+L_{w}\left(r_{0}\right) \int_{t_{1}}^{\infty} w_{1}\left(\tau, r_{0}\right) d \tau
$$

each such choice of $r_{0}$ and $t_{1}$ leads to a solution with a finite escape time. Defining

$$
\beta=\inf \left\{r_{0}: r\left(t ; t_{0}, r_{0}\right) \text { has a finite escape time }\right\},
$$

we see that $0<\alpha \leqq \beta<\infty$.

We now assert that the solution $\phi(t) \equiv r\left(t ; t_{0}, \alpha\right)$ exists on $\left[t_{0}, \infty\right)$ and that $\lim _{t \rightarrow \infty} \phi(t)=\infty$. To establish the first part of this statement, suppose that the maximal interval of existence of $\phi$ is $\left[t_{0}, T\right), t_{0}<T<\infty$. Choose $r_{1}>0$ and $t_{1}>T$ so that

$$
-G\left(r_{1}\right)<\int_{t_{1}}^{\infty} p_{2}(\tau) d \tau+L_{w}\left(r_{1}\right) \int_{t_{1}}^{\infty} w_{1}\left(\tau, r_{1}\right) d \tau
$$

As noted above, the solution $r\left(t ; t_{1}, r_{1}\right)$ has a finite escape time. Since the solutions of (1.1) depend continuously upon their initial data, there exists a bounded solution 
$r_{2}(t)=r\left(t ; t_{0}, r_{2}\right)$ such that $r_{2}(T) \geqq r_{1}$. This implies that $r_{2}\left(t_{1}\right) \geqq r_{1}=r\left(t_{1} ; t_{1}, r_{1}\right)$ which contradicts the fact that (1.1) has a unique solution to the initial value problem.

Next, we show that $\lim _{t \rightarrow \infty} \phi(t)=\infty$; for suppose that $\lim _{t \rightarrow \infty} \phi(t)=\phi_{\infty}$, $0<\phi_{\infty}<\infty$. Then, there exists a $T_{\infty} \geqq t_{0}$ such that

$$
2 \int_{T_{\infty}}^{\infty} w_{1}\left(\tau, 2 \phi_{\infty}\right) d \tau<L_{w}^{-1}\left(2 \phi_{\infty}\right) \int_{\phi_{\infty}}^{2 \phi_{\infty}} L_{w}(\rho) d \rho
$$

and

$$
2 \int_{T_{\infty}}^{\infty} w_{2}\left(\tau, \phi_{\infty}\right) d \tau<L_{w}^{-1}\left(\phi_{\infty}\right) \int_{\phi_{\infty}}^{2 \phi_{\infty}} L_{w}(\rho) d \rho .
$$

The solution $r\left(t ; T_{\infty}, \phi_{\infty}\right)$ is bounded above by $2 \phi_{\infty}$ and must exist at $t=t_{0}$. This is a contradiction and, therefore, $\lim _{t \rightarrow \infty} \phi(t)=\infty$.

An argument similar to that used in the previous paragraph shows that the solution $\psi(t) \equiv r\left(t ; t_{0}, \beta\right)$ exists on $\left[t_{0}, \infty\right)$. It follows immediately that $\lim _{t \rightarrow \infty} \psi(t)$ $=\infty$. This completes the proof of Theorem 1 .

REMARK 1. Condition (2.4) cannot be omitted in Theorem 1. This fact is illustrated by the following example.

EXAMPLE 1. The differential equation

$$
d r / d t=a(t) r, \quad r>0, t \geqq 0,
$$

where $a(t)$ is continuous and positive on $[0, \infty)$ and $\int^{\infty} a(\tau) d \tau<\infty$, satisfies all of the hypotheses of Theorem 1 except (2.4) if one takes $L_{w}(r)=r^{-2}, w_{1}(t, r)=0$, and $w_{2}(t, r)=a(t) r$. All of the solutions of $(3.4)$ are bounded on $[0, \infty)$ so that the solution space configuration is not that of Theorem 1 .

REMARK 2. A simple sufficient test for hypothesis (2.4) is the existence of the limit

$$
\lim _{r \rightarrow \infty} w_{2}(t, r) L_{w}(r)=w_{2}(t, \infty) L_{w}(\infty)>0 .
$$

In (2.4), $p_{2}(t)$ may be taken to be $w_{2}(t, \infty) L_{w}(\infty)$.

EXAMPLE 2. Let $a_{i}(t)(i=1,2)$ be continuous for $t \geqq 0, a_{1}(t)$ be positive and $a_{2}(t)$ be nonnegative on $[0, \infty)$, and

$$
\int^{\infty} a_{i}(t) d t<\infty, \quad i=1,2 .
$$

Suppose that $K_{i}(r)(i=1,2)$ are continuous for $r>0, K_{1}(r)$ is nondecreasing and $K_{2}(r)$ is nonincreasing on $(0, \infty)$, and $\int^{\infty}[1 / K(\rho)] d \rho<\infty$. The equation

$$
d r / d t=a_{1}(t) K_{1}(r)+a_{2}(t) K_{2}(r), \quad r>0, t \geqq 0,
$$

is not, in general, solvable in a closed form. However, Theorem 1 where $L_{w}(r)$ $=K_{1}^{-1}(r), \quad w_{1}(t, r) \equiv 0$, and $w_{2}(t, r)=a_{1}(t) K_{1}(r)+a_{2}(t) K_{2}(r)$ implies that this equation has positive bounded solutions. This is an example of an equation of the form (1.1) which could arise in comparison arguments but where $w(t, r)$ is not necessarily monotone in $r$. 
THEOREM 2. Let the hypotheses of Theorem 1 be satisfied. Suppose that $L_{w}(z)$ is differentiable, $L_{w}(1)=1$, and

$$
d L_{w}^{-1} / d z \geqq \xi>1
$$

for some constant $\xi$ and for all $z \geqq 1$. Furthermore, suppose that $w$ may be decomposed as $w(t, r)=f(t, r)+g(t, r)$ where $f$ and $g$ are nonnegative and $g(t, r) r^{-1}$ is nonincreasing in $r$ for $r \geqq 1$ and each fixed $t \geqq t_{0}$. Let $w$ be pseudo-submultiplicative with respect to $f, g$ and $L_{w}^{-1}$ in the sense that when $t \geqq t_{0}, x \geqq 1, y \geqq 1$ then

$$
w(t, x y) \geqq f(t, x) L_{w}^{-1}(y)+g(t, x) y-\sum_{i=1}^{n} a_{i}(t) P_{i}(y) Q_{i}(x),
$$

where $a_{i}(t), P_{i}(y), Q_{i}(x)$ are nonnegative, continuous functions of their respective variables

$$
\int^{\infty} a_{i}(\tau) d \tau<\infty, \quad i=1,2, \ldots, n
$$

and there exist constants $B_{i}, C_{i}$ such that

$$
Q_{i}(x) x^{-1} \leqq B_{i}, \quad i=1,2, \ldots, n, x \geqq 1,
$$

and

$$
P_{i}(y)(y-1)^{-1} \leqq C_{i}, \quad i=1,2, \ldots, n, y>1 .
$$

Then, in the conclusion of Theorem $1, \alpha=\beta$, that is, there is exactly one separatrix solution $\phi$ of (1.1) which exists on $\left[t_{0}, \infty\right)$ and satisfies $\lim _{t \rightarrow \infty} \phi(t)=\infty$.

It will be shown, following the proof of Theorem 2, that the hypotheses of Theorem 2 are sufficiently general to include a large class of differential equations of comparison type.

Proof of Theorem 2. By Theorem 1, there exists a solution $\phi$ of (1.1) such that $\lim _{t \rightarrow \infty} \phi(t)=\infty$. For convenience, it will be assumed that $\phi(t) \geqq 1$ for $t \geqq t_{0}$. First, we show that

$$
\int^{\infty} f(\tau, \phi(\tau)) \phi^{-1}(\tau) d \tau=\infty
$$

Equation (1.1) leads to the inequality

$$
\ln \phi(t) \phi^{-1}\left(t_{0}\right)-\int_{t_{0}}^{t} g(\tau, 1) d \tau \leqq \int_{t_{0}}^{t} f(\tau, \phi(\tau)) \phi^{-1}(\tau) d \tau .
$$

Since $\int^{\infty} g(\tau, 1) d t<\infty$ and $\lim _{t \rightarrow \infty} \phi(t)=\infty$, (3.6) follows from the above inequality.

Next, we will show that any solution $r(t)=r\left(t ; t_{0}, r_{0}\right)$ of (1.1) such that $r_{0}>\phi\left(t_{0}\right)$ $=\alpha$ has a finite escape time. If $z$ is defined by $z(t)=r(t) \phi^{-1}(t)$, then for all $t \geqq t_{0}$ for which $r(t)$ exists, $z(t)>1$. If $r(t)$ exists for all $t \geqq t_{0}$ then so does $z(t)$; suppose that $z(t)$ exists for all $t \geqq t_{0}$. The transformation $r(t)=\phi(t) z(t), t \geqq t_{0}$, applied to (1.1) leads to the equation

$$
z^{\prime}=w(t, \phi(t) z) / \phi(t)-w(t, \phi(t)) z / \phi(t), \quad z^{\prime}=d z / d t .
$$


Both $\phi(t)$ and $z(t)$ are not less than 1, so we may use the pseudo-submultiplicative property of $w$ to write

$$
z^{\prime} \geqq \frac{f(t, \phi(t))}{\phi(t)}\left[L_{w}^{-1}(z)-z\right]-\sum_{i=1}^{n} a_{i}(t) P_{i}(z) Q_{i}(\phi(t)) \phi^{-1}(t) .
$$

Next, we note that (3.5) implies that

$$
L_{w}^{-1}(z)-z \geqq(\xi-1)(z-1)
$$

for all $z \geqq 1$. Dividing both sides of (3.7) by $z(t)-1$, using (3.8), and integrating from $t_{0}$ to $t, t_{0}<t$, we obtain

$$
\begin{aligned}
\ln \frac{z(t)-1}{z\left(t_{0}\right)-1}+\sum_{i=1}^{n} \int_{t_{0}}^{t} a_{i}(\tau) P_{i}(z(\tau))(z(\tau)-1)^{-1} & Q_{i}(\phi(\tau)) \phi^{-1}(\tau) d \tau \\
& \geqq(\xi-1) \int_{t_{0}}^{t} f(\tau, \phi(\tau)) \phi^{-1}(\tau) d \tau .
\end{aligned}
$$

It follows from the hypothesis of the theorem that

$$
\begin{aligned}
\int_{t_{0}}^{\infty} a_{i}(\tau) P_{i}(z(\tau))(z(\tau)-1)^{-1} Q_{i}(\phi(\tau)) \phi^{-1}(\tau) d \tau & \\
& \leqq B_{i} C_{i} \int_{t_{0}}^{\infty} a_{i}(\tau) d \tau<\infty, \quad i=1,2, \ldots, n .
\end{aligned}
$$

This fact used in conjunction with (3.6) implies that $\lim _{t \rightarrow \infty}(z(t)-1)=\infty$; hence, $\lim _{t \rightarrow \infty} z(t)=\infty$.

Therefore, there exists an $\eta>1$ such that $z(t) \geqq \eta$ for all $t \geqq t_{0}$; hence, we can choose $\delta$ so that $\xi>\delta>1$ and $\eta>(\xi-1)(\xi-\delta)^{-1}>1$. Since $L_{w}^{-1}(z) \geqq \xi z-(\xi-1)$, it follows that

$$
L_{w}^{-1}(z)-z \geqq\left(1-\delta^{-1}\right) L_{w}^{-1}(z) .
$$

Dividing both sides of (3.7) by $L_{w}^{-1}(z)-z$ and then integrating leads to the inequality

$$
\begin{aligned}
\int_{z\left(t_{0}\right)}^{\infty}\left[L_{w}^{-1}(\zeta)-\zeta\right]^{-1} d \zeta \geqq & \int_{t_{0}}^{\infty} f(\tau, \phi(\tau)) \phi^{-1}(\tau) d \tau \\
& -\sum_{i=1}^{n} \int_{t_{0}}^{\infty} a_{i}(\tau) P_{i}(z(\tau))\left[L_{w}^{-1}(z(\tau))-z(\tau)\right]^{-1} Q_{i}(\phi(\tau)) \phi^{-1}(\tau) d \tau .
\end{aligned}
$$

The convergence of the last integrals above follows by noting (3.8) and an argument similar to that used to establish the convergence of the integrals in (3.10). Therefore, from (3.11), we obtain

$$
\int^{\infty} L_{w}(\rho) d \rho \geqq\left(1-\delta^{-1}\right) \int^{\infty}\left[L_{w}^{-1}(\zeta)-\zeta\right]^{-1} d \zeta=\infty .
$$


This contradicts hypothesis (3.2) and shows that $z(t)$, and hence $r(t)$, is not continuable to all $t \geqq t_{0}$. This completes the proof of Theorem 2 .

EXAMPLE 3. Let $m_{1} \leqq m_{2} \leqq \cdots \leqq m_{\mu} \leqq 1<n_{1} \leqq n_{2} \leqq \cdots \leqq n_{n}$. Consider the differential equation

$$
\frac{d r}{d t}=\sum_{i=1}^{n} b_{i}(t) r^{n_{i}}+\sum_{j=1}^{\mu} a_{j}(t) r^{m_{j}} \equiv w(t, r)
$$

In (3.12), $a_{i}$ and $b_{i}$ are continuous functions for $t \geqq 0 ; a_{j}(t) \geqq 0, \int^{\infty} a_{j}(\tau) d \tau<\infty$, $j=1,2, \ldots, m_{\mu} ; b_{i}(t) \geqq 0, b_{1}(t)>0$, and $\int^{\infty} b_{i}(\tau) d \tau<\infty, i=1,2, \ldots, n_{\eta}$.

We will now show that the solution space configuration of (3.12) is that indicated in Theorem 2; that is, there are bounded solutions, solutions with a finite escape time, and a single separatrix solution.

To see that the hypotheses of Theorem 2 are satisfied, we take $L_{w}(r)=r^{-n_{1}}$, $f(t, r)=\sum_{i=1}^{n} b_{i}(t) r^{n_{i}}$ and $g(t, r)=\sum_{j=1}^{\mu} a_{j}(t) r^{m_{j}}$. The only condition that is not clear is the pseudo-submultiplicative property. For $x \geqq 1, y \geqq 1, t \geqq 0$, we have

$$
\begin{aligned}
w(t, x y)= & {\left[\sum_{i=1}^{n} b_{i}(t) x^{n_{i}}\right] y^{n_{1}}+\left[\sum_{j=1}^{\mu} a_{j}(t) x^{m_{j}}\right] y } \\
& +\sum_{i=2}^{n} b_{i}(t) x^{n_{i}}\left[y^{n_{i}}-y^{n_{1}}\right]-\sum_{j=1}^{\mu} a_{j}(t) x^{m_{\jmath}}\left[y-y^{m_{j}}\right] \\
\geqq & f(t, x) L_{w}^{-1}(y)+g(t, x) y-\sum_{j=1}^{\mu} a_{j}(t) x^{m_{\jmath}}\left[y-y^{m_{j}}\right] .
\end{aligned}
$$

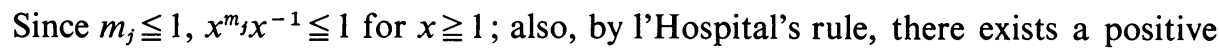
constant $B$ so that $\left(y-y^{m}\right) /(y-1) \leqq B, y>1$. Therefore, the hypotheses of Theorem 2 are satisfied and the solution space of (3.12) has a unique separatrix solution.

The remainder of this section investigates the three remaining types of solution set configurations as discussed in the introduction.

THEOREM 3. Let the hypotheses (2.1), (2.2), and (3.1) of Theorem 1 be satisfied; in addition, suppose that (2.3) is satisfied wherein $\int^{\infty} p_{1}(\tau) d \tau<\infty$ and

$$
\int^{\infty} L_{w}(\rho) d \rho=\infty
$$

Then, all solutions of (1.1) are bounded.

Proof. We define

$$
H(r)=\int_{0}^{r} L_{w}(\rho) d \rho
$$

where it will be assumed that $\int_{0} L_{w}(\rho) d \rho<\infty$; if this is not the case, then one may take any positive lower limit for the integral defining $H$. From (1.1), (2.1), (2.2) and (2.3) we obtain

$$
r\left(t ; t_{0}, r_{0}\right) \leqq H^{-1}\left[H\left(r_{0}\right)+\int_{t_{0}}^{\infty} p_{1}(\tau) d \tau+L_{w}\left(r_{0}\right) \int_{t_{0}}^{\infty} w_{2}\left(\tau, r_{0}\right) d \tau\right]
$$


This shows that all solutions of (1.1) are bounded and, hence, completes the proof of Theorem 3.

REMARK 3. A hypothesis that implies (2.3) is necessary. The differential equation

$$
d r / d t=a(t) r^{2}, \quad r>0, t \geqq 0,
$$

where $a(t)$ is continuous and positive on $[0, \infty)$, and $\int^{\infty} a(t) d t<\infty$, has unbounded solutions. But, one may satisfy all of the hypotheses of Theorem 3 except for (2.3) by taking $L_{w}(r)=1$.

The next two results will consider the case when

$$
\int^{\infty} w(\tau, \lambda) d \tau=\infty
$$

for all $\lambda>0$.

THEOREM 4. Let (2.1) and (3.2) be satisfied; furthermore, suppose that

$$
\int_{0}^{\infty} w_{1}(\tau, \lambda) d \tau=\infty
$$

for all $\lambda>0$. Then, no solution $r\left(t ; t_{0}, r_{0}\right), r_{0}>0, t_{0} \geqq 0$, is continuable to all $t \geqq t_{0}$.

Proof. Let $r\left(t ; t_{0}, r_{0}\right)$ be a solution which exists for all $t \geqq t_{0}$, then from (1.1) and (2.1), we have

$$
\begin{aligned}
\int_{r_{0}}^{\infty} L_{w}(\rho) d \rho & \geqq \int_{r_{0}}^{r\left(\infty ; t_{0}, r_{0}\right)} L_{w}(\rho) d \rho \\
& =\int_{t_{0}}^{\infty} w\left(\tau, r\left(\tau ; t_{0}, r_{0}\right)\right) L_{w}\left(r\left(\tau ; t_{0}, r_{0}\right)\right) d \tau \\
& \geqq L_{w}\left(r_{0}\right) \int_{t_{0}}^{\infty} w_{1}\left(\tau, r_{0}\right) d \tau=\infty
\end{aligned}
$$

This contradicts (3.2) and completes the proof of the theorem.

Next, we treat the case where (3.13) and (3.14) hold.

THEOREM 5. Let (2.3), (3.13), and (3.14) hold; then, all solutions $r\left(t ; t_{0}, r_{0}\right)$ of (1.1) exist on $\left[t_{0}, \infty\right)$ and $\lim _{t \rightarrow \infty} r\left(t ; t_{0}, r_{0}\right)=\infty$.

Proof. First, suppose that the solution $r\left(t ; t_{0}, r_{0}\right)$ exists only on $\left[t_{0}, T\right), t_{0}<T<\infty$. Then, we have

$$
\begin{aligned}
\infty & =\int_{r_{0}}^{\infty} L_{w}(\rho) d \rho=\int_{t_{0}}^{T} w\left(\tau, r\left(\tau ; t_{0}, r_{0}\right)\right) L_{w}\left(r\left(\tau ; t_{0}, r_{0}\right)\right) d \tau \\
& \leqq \int_{t_{0}}^{T} w_{1}\left(\tau, r\left(\tau ; t_{0}, r_{0}\right)\right) L_{w}\left(r\left(\tau ; t_{0}, r_{0}\right)\right) d \tau+L_{w}\left(r_{0}\right) \int_{t_{0}}^{T} w_{2}\left(\tau, r_{0}\right) d \tau \\
& \leqq \int_{t_{0}}^{T} p_{1}(\tau) d \tau+L_{w}\left(r_{0}\right) \int_{t_{0}}^{T} w_{2}\left(\tau, r_{0}\right) d \tau .
\end{aligned}
$$

Therefore, no solution has a finite escape time. 
Next, suppose that some solution $r\left(t ; t_{0}, r_{0}\right)$ is bounded on $\left[t_{0}, \infty\right)$; then,

$$
\begin{aligned}
\int_{r_{0}}^{r\left(\infty ; t_{0}, r_{0}\right)} L_{w}(\rho) d \rho & =\int_{t_{0}}^{\infty} w\left(\tau, r\left(\tau ; t_{0}, r_{0}\right)\right) L_{w}\left(r\left(\tau ; t_{0}, r_{0}\right)\right) d \tau \\
& \geqq L_{w}\left(r_{0}\right) \int_{t_{0}}^{\infty} w_{1}\left(\tau, r_{0}\right) d \tau+L_{w}\left(r\left(\infty ; t_{0}, r_{0}\right)\right) \int_{t_{0}}^{\infty} w_{2}\left(\tau, r\left(\infty ; t_{0}, r_{0}\right)\right) d \tau \\
& =\infty .
\end{aligned}
$$

This contradiction completes the proof of Theorem 5 .

REMARK 4. The example $d r / d t=r^{2}$ with $L_{w}(r)=1$ shows that the upper bounding function $p_{1}$ on $w_{1}(t, r) L_{w}(r)$ is, in some sense, a necessary requirement in Theorem 5 .

4. Results for equation (1.3). In this section we briefly consider equation (1.3) where $w$ is still assumed to satisfy the standing hypotheses from $\S 2$. The differential equation (1.3) may be transformed to the equation

$$
d s / d t=u(t, s)
$$

where $u(t, s)=s^{2} w\left(t, s^{-1}\right)$ by the substitution $r=s^{-1}$. Since (4.1) is of the form (1.1), the results of $\$ 3$ may be applied.

Note that the positive solutions of (1.3) are nonincreasing. Hence, solutions of (4.1) with a finite escape time correspond to solutions $r\left(t ; t_{0}, r_{0}\right), r_{0}>0$, of (1.3) for which there exists a $T_{0}>t_{0}$ such that $\lim _{t \rightarrow T_{0}^{-}} r\left(t ; t_{0}, r_{0}\right)=0$. Likewise, bounded solutions of (4.1) correspond to those solutions of (1.3) which are bounded away from zero. The unbounded solutions of (4.1) that are continuable to all $t \geqq t_{0}$ correspond to the solutions of (1.3) which have limit zero as $t$ approaches infinity. Thus, these three types of solutions of (1.3) may be studied by transforming (1.3) into (4.1) and then applying the results of $\S 3$.

Rather than state the analogues of the preceding theorems for the equation (1.3), we will consider a question raised by the first author in [3, Remark 1]. Is it possible to find an example of an equation of the form (1.3) with $w(t, r)$ nonincreasing in $r$, such that all solutions with a positive initial condition remain positive on a half line $\left[t_{0}, \infty\right)$ ? Under our hypotheses, the answer is no. To demonstrate this, we will apply Theorem 1 to the transformed equation (4.1). Since $w\left(t, s^{-1}\right)$ is nondecreasing in $s$, we can take $L_{w}(s)=s^{-2}$ and $w_{2} \equiv 0$ in the hypotheses of Theorem 1 . Therefore, if (3.1) is satisfied, the question is answered. If (3.1) is not satisfied, it is known that the equation (1.3) cannot have the desired property [3, Remark 2].

\section{REFERENCES}

1. Fred Brauer, Bounds for solutions of ordinary differential equations, Proc. Amer. Math. Soc. 14 (1963), 36-43. MR 26 \#397.

2. Fred Brauer and J. S. W. Wong, On asymptotic behavior of perturbed linear systems, J. Differential Equations 6 (1969), 142-153. MR 39 \#570.

3. Thomas G. Hallam, The asymptotic manifold of a perturbed linear differential equation as determined by the comparison principle, Ann. Mat. Pura Appl. 87 (1970), 299-313. 
4. T. G. Hallam and J. W. Heidel, The asymptotic manifolds of a perturbed linear system of differential equations, Trans. Amer. Math. Soc. 149 (1970), 233-241. MR 41 \#2136.

5. Philip Hartman, On an ordinary differential equation involving a convex function, Trans. Amer. Math. Soc. 146 (1969), 179-202.

6. J. W. Heidel, Global asymptotic stability of a generalized Liénard equation, SIAM J. Appl. Math. 19 (1970), 629-636.

7. - Periodic solutions of $x^{\prime \prime}+f(x)\left(x^{\prime}\right)^{2 n}+g(x)=0$ with arbitrarily large period, Ann. Polon. Math. 24 (1971), 351-356.

8. I. I. Kolodner, J. Lehner and G. M. Wing, On first order ordinary differential equations arising in diffusion problems, J. Soc. Indust. Appl. Math. 12 (1964), 249-269. MR 29 \#4946.

9. G. Ladas, V. Lakshmikantham and S. Leela, On the perturbability of the asymptotic manifold of a perturbed system of differential equations, Proc. Amer. Math. Soc. 27 (1971), 65-71.

10. V. Lakshmikantham and S. Leela, Differential and integral inequalities. Vol. I, Academic Press, New York, 1969.

11. T. Yoshizawa, Stability theory by Liapunov's second method, Publ. Math. Soc. Japan, no. 9, Math. Soc. Japan, Tokyo, 1966. MR 34 \#7896.

\author{
Florida State University, \\ TAllahassee, Florida 32306 \\ UNIVERSITY OF TENNESSEE, \\ KNoXville, TenNessee 37916
}

